\title{
Linear Transformations on Two Dimensions Delays Differential Equations Preserving Dynamics
}

\author{
Lin Deng, Feng Zhang, Jinli Xu \\ Department of mathematics, Northeast Forestry University, Harbin, China
}

*Corresponding Author: Lin Deng, Department of mathematics, Northeast Forestry University, Harbin, China

Abstract: Let $R$ be the real field. We consider the two dimension systems: $\dot{x}(t)=A x(t)+B x(t-\tau)$

where $A, B \in R^{2 \times 2}, \tau>0$. The characteristic polynomial of above system is $\operatorname{det}\left(\lambda I-A-B e^{-\lambda \tau}\right)$, we determine the form of linear map $\phi: R^{2 \times 2} \rightarrow R^{2 \times 2}$ preserving the characteristic polynomial.

Keywords: delays differential equations, linear preserver problem, linear map

\section{INTRODUCTION}

Let $R$ be the real field. We consider the two dimension systems:

$\dot{x}(t)=A x(t)+B x(t-\tau)$

where $A, B \in R^{2 \times 2}, \tau>0$. The characteristic polynomial of (1) is

$\operatorname{det}\left(\lambda I-A-B e^{-\lambda \tau}\right)$

In this paper, we determine the form of linear map $\phi: R^{2 \times 2} \rightarrow R^{2 \times 2}$ preserving the characteristic equation of (1).

Theorem 1. ([1,2,3]) Suppose $\phi: R^{n \times n} \rightarrow R^{n \times n}$ is a linear map. Then

$\operatorname{det}(\lambda I-A)=\operatorname{det}(\lambda I-\phi(A))$ for all $A \in R^{n \times n}$

if and only if $\phi$ is of the form

$X \mapsto P X P^{-1}$, or $X \mapsto P X^{T} P^{-1}, \forall X \in R^{n \times n}$

where $P$ is a nonsingular matrix.

Theorem 2. Suppose $\phi: R^{n \times n} \rightarrow R^{n \times n}$ is a linear map. Then

$\operatorname{det}\left(\lambda I-B e^{-\lambda \tau}\right)=\operatorname{det}\left(\lambda I-\phi(B) e^{-\lambda \tau}\right)$ for all $B \in R^{n \times n}$

if and only if $\phi$ is of the form

$X \mapsto P X P^{-1}$, or $X \mapsto P X^{T} P^{-1}, \forall X \in R^{n \times n}$

where $P$ is a nonsingular matrix.

Proof. Let $E_{r}(X)$ is the sum of all principal $r \times r$ sub determinants of $X$. It is easy to see

$\operatorname{det}\left(\lambda I-B e^{-\lambda \tau}\right)=\Sigma_{r}(-1)^{r} E_{r}(B) \lambda^{n-r} e^{-r \lambda \tau}$.

By $\operatorname{det}\left(\lambda I-B e^{-\lambda \tau}\right)=\operatorname{det}\left(\lambda I-\phi(B) e^{-\lambda \tau}\right)$, we obtain $E_{r}(B)=E_{r}(\phi(B))$. Hence

$\operatorname{det}(\lambda I-B)=\Sigma_{r}(-1)^{r} E_{r}(B) \lambda^{n-r}=\Sigma_{r}(-1)^{r} E_{r}(\phi(B)) \lambda^{n-r}=\operatorname{det}(\lambda I-\phi(B))$,

That is $\phi$ preserving ordinary characteristic polynomial. 
Theorem 3. Suppose $\phi: R^{n \times n} \rightarrow R^{n \times n}$ is a linear map and $B \in R^{n \times n}$ is nonzero matrix. Then

$$
\operatorname{det}\left(\lambda I-A-B e^{-\lambda \tau}\right)=\operatorname{det}\left(\lambda I-\phi(A)-\phi(B) e^{-\lambda \tau}\right) \text { for all } A \in R^{n \times n}
$$

if and only if $\phi$ is of the form

$$
X \mapsto P X P^{-1} \text {, or } X \mapsto P X^{T} P^{-1}, \forall X \in R^{n \times n}
$$

where $P$ is a nonsingular matrix.

Proof. Setting $A=0$, we obtain

$$
\operatorname{det}\left(\lambda I-B e^{-\lambda \tau}\right)=\operatorname{det}\left(\lambda I-\phi(B) e^{-\lambda \tau}\right) \text {. }
$$

Hence, $B$ and $\phi(B)$ have the same characteristic polynomial, so are the eigenvalues. Without loss of generality, we may assume that $B$ and $\phi(B)$ are already in their canonical form.

We next assume $n=2$.

Case 1. $B$ has mutually different eigenvalues. In this case, $B$ and $\phi(B)$ has the common canonical form, say $B=\phi(B)=b_{1} \oplus b_{2}$. We assume $\phi\left(E_{11}\right)=\left[\begin{array}{ll}x_{11} & x_{12} \\ x_{21} & x_{22}\end{array}\right]$ in each determine. By

$\operatorname{det}\left[\begin{array}{cc}\lambda-b_{1} e^{-\lambda \tau}-1 & 0 \\ 0 & \lambda-b_{2} e^{-\lambda \tau}\end{array}\right]=\operatorname{det}\left[\begin{array}{cc}\lambda-b_{1} e^{-\lambda \tau}-x_{11} & -x_{12} \\ -x_{21} & \lambda-b_{2} e^{-\lambda \tau}-x_{22}\end{array}\right]$

that is

$$
\begin{aligned}
\left(\lambda-b_{1} e^{-\lambda \tau}-1\right)\left(\lambda-b_{2} e^{-\lambda \tau}\right) & =\lambda^{2}-\lambda-\left(b_{1}+b_{2}\right) \lambda e^{-\lambda \tau}+b_{2} e^{-\lambda \tau}+b_{1} b_{2} e^{-2 \lambda \tau} \\
& =\lambda^{2}-\left(x_{11}+x_{22}\right) \lambda-\left(b_{1}+b_{2}\right) \lambda e^{-\lambda \tau}+\left(x_{22} b_{1}+x_{11} b_{2}\right) e^{-\lambda \tau} \\
& +b_{1} b_{2} e^{-2 \lambda \tau}+x_{11} x_{22}-x_{12} x_{21}
\end{aligned}
$$

Hence,

$$
\left(x_{11}+x_{22}-1\right) \lambda+\left(x_{22} b_{1}+x_{11} b_{2}-b_{2}\right) e^{-\lambda \tau}+\left(x_{11} x_{22}-x_{12} x_{21}\right)=0
$$

We have $x_{11}=1, x_{22}=0$ and $x_{12} x_{21}=0$. Similarly, we can obtain $\phi\left(E_{22}\right)=\left[\begin{array}{cc}0 & y_{12} \\ y_{21} & 1\end{array}\right]$, with $y_{12} y_{21}=0 . \phi\left(E_{12}\right)=\left[\begin{array}{cc}0 & z_{12} \\ z_{21} & 0\end{array}\right]$, with $z_{12} z_{21}=0, \phi\left(\boldsymbol{E}_{21}\right)=\left[\begin{array}{cc}\mathbf{O} & w_{12} \\ w_{21} & 0\end{array}\right]$, with $w_{12} w_{21}=0$. We assume $z_{12} \neq 0$, then $z_{21}=0$. It is easy to see $w_{12}=0$, and $w_{21} \neq 0$, and $z_{12} w_{21}=1$. Thus, we can obtain $x_{12}=0, x_{21}=0, y_{12}=0, y_{21}=0$, hence, $\phi\left(E_{11}\right)=E_{11}$, and $\phi\left(E_{22}\right)=E_{22}$. Let

$P=1 \oplus z_{12}^{-1}$, then $\phi(X)=P X P^{-1}$.

Case 2. $B=\mu I_{2}$. Then $\phi(B)=\mu I_{2}$, or $\phi(B)=\mu I_{2}+E_{12}$.

Subcase I. $B=\phi(B)=\mu I_{2}$. Similar as Case 1, we can see $\operatorname{det} \phi\left(E_{11}\right)=0$, and $\operatorname{tr} \phi\left(E_{11}\right)=1$, without loss of generality, we can assume $\phi\left(E_{11}\right)=E_{11}$. By $B=\phi(B)=\mu I_{2}$, we can obtain $\phi\left(E_{22}\right)=E_{22}$. Using the similar method as Case 1, we see the result holds.

Subcase II. $B=\mu I_{2}$ and $\phi(B)=\mu I_{2}+E_{12}$. we will prove this case cannot appear.

Noting that

$\operatorname{det}\left[\begin{array}{cc}\lambda-\mu e^{-\lambda \tau}-a & -b \\ -c & \lambda-\mu e^{-\lambda \tau}-d\end{array}\right]=\operatorname{det}\left[\begin{array}{cc}\lambda-\mu e^{-\lambda \tau}-x & e^{-\lambda \tau}-y \\ -z & \lambda-\mu e^{-\lambda \tau}-u\end{array}\right]$

Hence $\lambda^{2}-(a+d) \lambda+(a+d-2) \mu e^{-\lambda \tau}+\mu^{2} e^{-2 \lambda \tau}+a d-b c$ and $\lambda^{2}-(x+u) \lambda+(x+u-2) \mu e^{-\lambda \tau}+z e^{-\lambda \tau}+\mu^{2} e^{-2 \lambda \tau}+x u-y z$. Thus $a+d=x+u$, 
$(a+d-2) \mu=(x+u-2) \mu+z, a d-b c=x u-y z$.

This implies $z=0$, i.e. $\phi\left(M_{2}\right) \subset T_{2}$ (the up triangle matrix set), and $\operatorname{det} X=\operatorname{det} \phi(X)$, which is a contradiction.

Case III. $\boldsymbol{B}=\boldsymbol{\mu} \boldsymbol{I}_{2}+\boldsymbol{E}_{12}$, similar to the above, and then we complete the proof.

\section{REFERENCES}

[1] C. K. Li and S. Pierce, A survey on linear preserver problems, Amer. Math. Monthly, vol. 108, pp. 591-605, 2001.

[2] M. H. Chan, M. H. Lim and K. K. Tan, Linear preservers on matrices, Linear Algebra and its Applications, vol. 93, pp. 67-80, 1987.

[3] M. Marcus and B. N. Moyls, Linear transformtions on algebras of matrices, Canad. J. Math., vol. 61, pp. 6166, 1959.

Citation: Deng, L.et.al, (2019). Linear Transformations on Two Dimensions Delays Differential Equations Preserving Dynamics. International Journal of Scientific and Innovative Mathematical Research (IJSIMR), 7(1), pp.1-3. http://dx.doi.org/10.20431/2347-3142.0701001

Copyright: (C) 2019 Authors, This is an open-access article distributed under the terms of the Creative Commons Attribution License, which permits unrestricted use, distribution, and reproduction in any medium, provided the original author and source are credited. 